\title{
La neurostimolazione midollare nel trattamento dell'ischemia critica non rivascolarizzabile del paziente uremico
}

\author{
U. Brummer ${ }^{1}$, P. Cappelli ${ }^{1}$, A. Piano ${ }^{1}$, A. Cardillo ${ }^{1}$, A. Perilli², M. Bonomini ${ }^{1}$ \\ IU.O. di Nefrologia e di Terapia Emodepurativa, Ospedale Clinicizzato "SS. Annunziata", Università "G. \\ D'Annunzio", Chieti \\ ¿U.O. di N efrologia, Ospedale "M aria SS. dello Splendore", Giulianova
}

\section{Caso Clinico}

Un paziente maschio di 66 anni, affetto da insufficienza renale cronica secondaria a nefropatia diabetica e in trattamento emodialitico sostitutivo dall'ottobre 2002, è stato ricoverato presso il nostro Centro per la presenza di claudicatio intermittens con autonomia di marcia inferiore a 200 metri e la recente comparsa di dolore a riposo durante le ore notturne in corrispondenza della gamba sinistra. Le caratteristiche del trattamento emodialitico al momento del ricovero erano le seguenti: tampone bicarbonato, ritmo trisettimanale, durata della seduta di 4 ore, membrana in polisulfone con superficie di $1.8 \mathrm{~m}^{2}$; terapia intradialitica: Paracalcitolo $5 \mu \mathrm{g}$ e.v. alla settimana, Eritropoietina beta 4000 UI s.c. $x$ $3 /$ sett., Sodio fer rigluconato $62.5 \mathrm{mg}$ e.v alla settimana. La terapia domiciliare comprendeva Paracetamolo 1 $\mathrm{g} /$ die, Tramadolo SR100 $1 \mathrm{cpr} /$ die, Atorvastatina 10 $\mathrm{mg} 1 \mathrm{cpr} /$ die, Lantanum carbonato $750 \mathrm{mg} 1 \mathrm{cpr}$ a pranzo e a cena, Lansoprazolo $15 \mathrm{mg} 1 \mathrm{cpr} / \mathrm{die}$, insulina Lantus e N ovorapid secondo lo schema stabilito dal Centro Antidiabetico di riferimento.

L'esame obiettivo rilevava un'ischemia critica dell'ar to inferiore sinistro (stadio IVa secondo la classificazione di Leriche-F ontaine, Tab. I), caratterizzata da pallore cutaneo dell'arto inferiore sinistro con polsi periferici presenti e validi, e omolateralmente presenza di piccola lesione ischemica in corrispondenza del primo dito. Esami ematochimici eseguiti al momento del ricovero documentavano: $\mathrm{Hb} 11.8 \mathrm{~g} / \mathrm{dL}, \mathrm{GB}$ 12050/ mmc, emoglobina glicosilata $6.7 \%$ fibrinogeno $590 \mathrm{mg} \%$ PCR 8.5 $\mathrm{mg} \%$ (valori normali $<0.5$ ), elettroforesi sierica nella norma, PTH $273 \mathrm{pg} / \mathrm{mL}$, calcemia $8.36 \mathrm{mg} \%$ fosforemia 6.53 $\mathrm{mg} \%$ colesterolo totale $178 \mathrm{mg} \%$ colesterolo LDL 107 $\mathrm{mg} \%$ trigliceridi $131 \mathrm{mg} \% \mathrm{~N}$ el sospetto di una osteomie-

TABELLA I - CLASSIFICAZIONE DELL'ISCHEMIA CRONICA DEGLI ARTI INFERIORI SECONDO LERICHE-FONTAINE

\begin{tabular}{ll} 
Stadio Sintomi e segni \\
\hline I & $\begin{array}{l}\text { Asintomatico } \\
\text { Claudicatio intermittente senza dolore a riposo } \\
\text { Ila: Autonomia di marcia }>200 \mathrm{~m}\end{array}$ \\
III $\quad \begin{array}{l}\text { Claudicatio e dolore a riposo, assenza di alterazioni } \\
\text { cutanee }\end{array}$ \\
IV Stadio III +alterazioni cutanee (necrosi/ gangrena) \\
IVa: infiammazione locale \\
IVb: infiammazione estesa
\end{tabular}


lite della prima falange del primo dito del piede sinistro abbiamo sottoposto il paziente a un $\mathrm{Rx}$ del piede sinistro che evidenziava un riassorbimento dell'apofisi ungueale della falange del primo dito, diffusa osteopenia e ateromasia parietale calcifica dei vasi loco-regionali. Un esame ecocolorD oppler arterioso degli arti inferiori segnalava bilateralmente segmenti iliaco-femorali comuni, biforcazione femorale, femorale profonda e femorale superficiale di dimensioni nella norma con modeste alterazioni calcifiche miointimali, senza evidenza di lesioni emodinamicamente significative; piccole placche fibrocalcifiche delle poplitee, a sinistra flusso demodulato sulle tibiali, a destra flusso diretto sulle tibiali. Un esame angio-TC degli arti inferiori, effettuato successivamente, evidenziava diffusa ateromasia parietale, in parte calcifica, dell'aor ta toraco-addominale; pervi e di buon calibro gli assi iliaco-femorali bilateralmente; filiformi i vasi della gamba con evidenza di stenosi multiple del tratto medio-distale della tibiale anteriore bilateralmente.

Alla luce degli esami strumentali i chirurghi vascolari non ritenevano opportuno effettuare un inter vento di rivascolarizzazione o di angioplastica. II paziente, essendo affetto da angiodisplasia intestinale (documentata precedentemente), non poteva essere sottoposto a un ciclo di terapia con prostaglandine. N ell'intento di trattare efficacemente il dolore ed, eventualmente, la microcircolazione del piede, stabilizzando la lesione ischemica, abbiamo sottoposto il paziente a impianto di elettrostimolatore midollare. In anestesia locale il conduttore dell'elettrostimolatore midollare (SCS) veniva posizionato nello spazio epidurale dei dermatomeri interessati e regolato sino a quando il paziente riferiva parestesie che si estendevano a tutta l'area dolente dell'ar to. II generatore di impulsi veniva impiantato in una tasca sottocutanea del fianco. Nel periodo di follow-up abbiamo assistito a un miglioramento significativo della sintomatologia dolorosa, non sono comparse nuove lesioni cutanee e a distanza di un anno il paziente è stato sottoposto ad amputazione del primo dito del piede sinistro.

\section{Discussione}

La malattia vascolare periferica è un'alterazione piuttosto comune nei pazienti affetti da insufficienza renale cronica terminale (ESRD). La sua prevalenza secondo i dati dello studio DOPPS ( 1 ) è del $25.3 \%$ con oscillazioni che variano tra il $12 \%$ e il $38 \%$ Inoltre è associata a un'elevata mor talità e morbilità. Studi preliminari suggeriscono che non solo fattori di rischio tradizionali come età avanzata, ipertensione, iperlipidemia, fumo, diabete mellito e malattia coronarica contribuiscono all'aumentata morbilità e mortalità cardiovascolare nei pazienti con ESRD, ma anche fattori non tradizionali come l'iperparatiroidismo, lo stato infiammatorio cronico, i livelli di apolipoproteina (a) e iperomocisteinemia potrebbero avere un ruolo importante nello sviluppo e nella progressione dell'arteriopatia obliterante cronica periferica (AOCP) in questi pazienti (2-4).

Nei pazienti con ischemia critica agli arti inferiori, sia procedure che tendono a conser vare l'ar to, come la ricostruzione vascolare e l'angioplastica transluminale percutanea sia terapie non chirurgiche, come l'infusione endovenosa di prostaglandine, sono considerate trattamenti di scelta. Sfortunatamente il 3-5\% dei pazienti emodializzati non è passibile di un inter vento primario, di un reinter vento o di terapia farmacologica e viene sottoposto ad amputazione a carico degli arti inferiori (5, 6). In questi pazienti, per i quali il controllo del dolore è prioritario, la SCS potrebbe essere considerata un'ultima risorsa prima dell'amputazione. La stimolazione del midollo spinale (Spinal Cord Stimulation, SCS) è una procedura neuromodulatoria che rappresenta un trattamento alternativo per quei pazienti affetti da AOCP non idonei a procedure di rivascolarizzazione. O biettivi della SCS sono la diminuzione del dolore, la prevenzione o il dilazionamento dell'amputazione e il miglioramento della qualità di vita.

Poiché la popolazione in dialisi è in genere affetta da multiple comorbidità, è importante mantenere una ragionevole qualità di vita legata alla salute.

Korzets et al (7) suggerivano che il dolore è un fattore molto rilevante nel valutare la qualità di vita legata alla salute del paziente. Pertanto la gestione del dolore dovrebbe essere parte integrante della qualità delle attenzioni che vanno for nite al paziente. II trattamento del dolore ischemico cronico attraverso la SCS è stato introdotto da Cook et al (8) nel 1973. Questo e altri studi riportano un eccellente controllo del dolore, l'aumento della guarigione delle ulcere, la diminuzione del bisogno di analgesici per via orale e un miglioramento nelle attività della vita quotidiana $(9,10)$. Gli effetti benefici della SCS sembrano legati apparentemente a un incremento del flusso sanguigno a livello dell'arto ischemico. I meccanismi attraverso cui la neurostimolazione aumenta la microcircolazione non sono ancora completamente conosciuti. Varie teorie sono state proposte: vasodilatazione mediata dal rilascio locale di sostanze vasoattive attraverso fibre afferenti di piccolo diametro nelle radici e nelle colonne dorsali; vaso- dilatazione mediata indirettamente dai centri vasoregolatori del midollo; mediazione della vasodilatazione da parte del SNA sul midollo spinale con rilascio da parte delle radici dorsa- 
li di un vasodilatatore neurormonale chiamato peptide cor relato geneticamente alla calcitonina (calcitonin gene-related peptide) e con attenuazione del rilascio di aminoacidi eccitatori da parte delle corna dorsali con meccanismo GABA mediato (11-14).

$\mathrm{Nel}$ nostro paziente un sollievo dal dolore attribuibile alla SCS è suggerito dalla significativa riduzione nell'uso di presidi analgesici. Ci sono pochi dati obiettivi sulla gestione farmacologica del dolore nei pazienti in emodialisi. Davison (15) osser vava un'insufficiente soppressione del dolore non neoplastico in pazienti con IRC a dispetto della somministrazione di forti dosaggi di oppioidi. Prima dell'impianto del dispositivo il paziente assumeva una combinazione di analgesici maggiori o minori. Nonostante questo la soppressione del dolore era inadeguata. La farmacocinetica degli oppioidi nei pazienti con ESRD è complessa; gli effetti collaterali più comunemente sperimentati sono confusione, mioclono, nausea, vomito, sedazione e depressione respiratoria. In più i pazienti che utilizzano in combinazione aspirina, FANS e anticoagulanti sono a rischio di sanguinamenti gastrointestinali.

Cosi come ogni procedura invasiva l'impianto di un dispositivo di SCS comporta cer ti rischi. Complicanze riportate sono il non funzionamento $(3 \% 4 \%)$, infezioni ( $3 \% 5 \%$ ), dislocazione e rottura (11\%36\%), perdita di liquido cerebrospinale ( $1 \%$ ) e meningite (0.5\%) (16). La complicanza più temibile è il danno neurologico causato da un danno intraoperatorio delle corna o delle radici spinali o dalla successiva compressione da parte di un coagulo intraspinale o di un ematoma epidurale. Noi non abbiamo osservato alcuna complicanza causata dalla SCS.

In conclusione, a nostro parere, l'impianto di un dispo- sitivo SCS da parte di un chirurgo d'esperienza, in pazienti con ESRD affetti da ischemia critica degli arti inferiori e non altrimenti trattabili, può migliorare notevolmente la qualità di vita e alleviare il dolore, giustificando i costi del dispositivo.

\section{TEST DI VE RIFICA - 1}

1) Qual è la prevalenza, secondo lo studio DOPPS, della malattia vascolare periferica?
a) $50 \%$
b) $25 \%$
c) $75 \%$
d) $5 \%$

2) Qual è la complicanza più temibile dell'impianto di un dispositivo di SCS?
a) non funzionamento
b) meningite
c) dislocazione e rottura
d) danno delle corna o delle radici spinali

3) Nei pazienti con ischemia critica degli arti inferiori quali, tra i seguenti, sono considerati trattamenti di scelta?
a) ricostruzione vascolare
b) angioplastica transluminale percutanea
c) infusione endovenosa di prostaglandine
d) tutte le precedenti

La risposta corretta alle domande è a pag. 42

e-mail: u.brummer@aslchieti.it

\section{B ibliografia}

1. Rajagopalan S, Dellegrottaglie S, Furniss AL, et al. Peripheral arterial disease in patients with end-stage renal disease: observations from the dialysis outcomes and practice patterns study (DOPPS). Circulation 2006; 114: 1914-22.

2. Nishizawa Y, Shoji T, Kawagishi T, M orii H. Atherosclerosis in uremia: possible roles of hyperparathyroidism and intermediate density lipoprotein accumulation. Kidney Int 2000; 62 (Suppl): S90-2.
3. Yeun JY, Levine RA, M antadilok V, Kaysen GA. C-reactive protein predicts all-cause and cardiovascular mortality in hemodialysis patients. Am J Kidney Dis 2000; 35: 469-76.

4. Bachman J, Tepel M, Raidt $\mathrm{H}$, et al. Hyperhomocysteinemia and the risk for cardiovascular disease. J Am Soc Nephrol 1995; 6: 121-5.

5. Skevington SM. Investigating the relationship between pain and discomfort and quality of life using the WHOQ OL. Pain 1998; 76: 395-406.

6. Eggers PW, Gohdes D, Pugh J. Nontraumatic lower 
extremity amputations in the Medicare end-stage renal disease population. Kidney Int 1999; 56: 152433.

7. Korzets A, Ori $Y$, Rathaus $M$, et al. Extremity amputations in chronically dialysed patients: A 10 year study. IMAJ 2003; 5: 501-5.

8. Cook AW, Oygar A, Baggenstos P, Pacheco S, Klerig E. Vascular disease of extremities. Electrical stimulation of spinal cord and posterior roots. N Y State J M ed 1976; 76: 366-8.

9. Ubbink DT, Vermeulen $H$, Spincemaille $G H$, Gersbach PA, Berg P, Amann W. Systematic review and metaanalysis of controlled trails assessing spinal cord stimulation for inoperable critical leg ischaemia. $\mathrm{Br} J$ Surg 2004; 91: 948-55.

10. Brümmer $U$, Condini V, Cappelli $P$, et al. Spinal cord stimulation in hemodialysis patients with critical lowerlimb ischemia. Am J Kidney Dis 2006; 47: 842-7.

11. Hilton $\mathrm{S}, \mathrm{M}$ arshall J. Dorsal root vasodilatation in cat skeletal muscle. J Physiol 1980; 299: 277-88.
12. Linderoth B, Fedorcsak I, Meyerson B. Peripheral vasodilatation after spinal cord stimulation: animal studies of putative effector mechanisms. Neurosurgery 1991; 28: 187-95.

13. Linderoth B, Herregodts $P$, M eyerson B. Sympathetic medication of peripheral vasodilatation induced by spinal cord stimulation: animal studies of the role of cholinergic and adrenergic receptor subtypes. Neurosurgery 1994; 35: 711-9.

14. Cui JG, O 'Connor WT, Ungerstedt U, Linderoth B, M eyerson B. Spinal cord stimulation attenuates augmented dorsal horn release of excitatory amino acids in mononeuropathy via a GABAergic mechanism. Pain 1997; 73: 87-95.

15. Davison SN. Pain in hemodialysis patients: Prevalence, Cause, Severity, and Management. Am J Kidney Dis 2003; 42: 1239-47.

16. Klomp HM, Spincemaille GH, Steyerberg EW, et al. Spinal cord stimulation in critical limb ischaemia: a randomized trial. Lancet 1999; 353: 1040-104. 